\title{
A Generic Description Model for the Structure of Atomic Nucleus with New Interpretation of the Strong Forces
}

\author{
Hongguang Yang, Weidong Yang \\ Munich, Germany \\ Email: hyang2013@gmail.com,wdyconf@gmail.com
}

How to cite this paper: Yang, H.G. and Yang, W.D. (2020) A Generic Description Model for the Structure of Atomic Nucleus with New Interpretation of the Strong Forces. Journal of Modern Physics, 11, 1132-1156. https://doi.org/10.4236/jmp.2020.118071

Received: June 24, 2020

Accepted: August 3, 2020

Published: August 6, 2020

Copyright $\odot 2020$ by author(s) and Scientific Research Publishing Inc. This work is licensed under the Creative Commons Attribution International License (CC BY 4.0).

http://creativecommons.org/licenses/by/4.0/

\section{(c) (i) Open Access}

\begin{abstract}
The present investigation is motivated by finding and developing an easily understandable solution in the context of unified quantum and gravitational theories. Model-based methods are applied, with emphasis on structural descriptions by introducing some strong hypotheses. A subset of the introduced hypotheses led to a surprising understanding of the internal structure and construction of quarks, neutrons, protons and more complex atomic nuclei. The research work therefore focused mainly on the model-based interpretation of subatomic processes. The results obtained so far and presented in this paper are new. They consist of a generic description model for the structure of atomic nuclei. This model contains two important structural links that originate from the initial phase of the cosmological big bang. They hold atomic parts together and are involved in many known nuclear fusion and fission processes. Modifications of them, including the electron-positron annihilation process, are necessary and will be described. A new interpretation of the strong forces from the Standard Model is possible and will be given. In addition, the formation processes for electron and positron particles are considered. Based on the structural relationships, a deeper understanding of matter transformations (transmutations), early cosmological processes and dark matter has been achieved. All challenges of this work are the logical conclusions from the used hypotheses on two structural links. They need to be further investigated and verified by theoretical and experimental works. The postulated particle in this paper, as accompanying product in the electron-positron annihilation, will play a major role for the future investigations.
\end{abstract}

\section{Keywords}

Atomic Kernel Structure, Reinterpretation of Strong Forces, Kernel Structure of Hydrogen and Its Isotopes, Kernel Structure of Helium and Its Isotope, 
Stellar Nuclear Fusion, Nuclear Fission, Double Space Link, Triple Space

Link, Matter Lifecycle in the Context of Cosmology

\section{Introduction}

The commonly used methods for the analysis of subatomic processes from the well-known Standard Model and the Quantum Chromodynamics Model QCD focus mainly on elementary particles and their interactions with each other (Ref. [1] [2] [3] [4]). Recent experimental data provide different aspects of our understanding of strong forces for the atom core structure (Ref. [5]-[10]). More systematic research on the structural make-up of atomic nuclei, e.g. how neutrons and protons are geometrically arranged within a nucleus, can help to improve the analysis and understanding of subatomic processes and macro-physical aspects.

The generic description model, the novelty of this work-we call it the YY model-aims at this question and can help to achieve improvements. The starting point, unlike what we usually do to explore the quantum nature of atomic and subatomic physics, is the introduction of two essential structural instances that combine all materialized quarks to form an atomic nucleus. These are the "Pairing Space Link PSL" and the "Triple Space Link TSL". The first, PSL, allows a new description of quarks, neutrons and protons in a deterministic spatial structure (Sections 2 and 3). The second, TSL, extends the design scheme to build complex atomic nuclei and isotopes, as applied for deuterium, tritium and helium (Sections 4, 5, 6, 7 and 8).

"Yin" and "Yang", which represent conceptual units of one third of an electric charge, negative and positive respectively, are defined. Hypothetically, a pair of them forms a PSL, while three PSLs form a TSL. The common node must always be "triple charged" for a complete positive or negative electrical unit. In addition, and as a rule, the numbers of Yin's and Yang's must be preserved in all nuclear transformations.

The hypotheses are not proven. However, their effects are clear and easy to understand. The YY model based on PSL/TSL allows a deterministic view of all the building quarks within or around a nucleus and their disassembly and reassembly in all nuclear transformation scenarios. Electric charges also include the inner structural instances. The internal charge balance effect (ICB, Sections 8 and 11), which effectively replaces, or stands for the QCD mechanism, allows a new interpretation of the strong forces of the Standard Model. As a result, the interaction mechanism of QCD could be simplified or reduced to those for PSL and TSL. The logical conclusion is a well-defined modification of our understanding of the electron, positron and their annihilation process (Sections 9 and $10)$.

Taking into account known facts about kernel construction, design rules for the YY model are introduced (Section 11). We were able to explain some 
well-researched processes of stellar nuclear fusion and predict a few unknown aspects that play a role in these processes (Section 12). Furthermore, the involvement of TSLs in nuclear fission is considered (Sections 13).

Using the YY model, more structural details will be visible for an atomic nucleus than is the case with the conventional description. It allows very fine considerations of the spatial distribution of protons and neutrons within or around an atomic nucleus-as we will see, this distribution is a matter of their up and down quarks.

In connection with TSL, a new "Y-particle" is postulated, which plays an important role in the energy-matter life cycle around nuclear fusion and fission processes, in electron-positron annihilation and in the formation of matter during the inflation phase of the big bang. Based on the predicted origin of TSL, a rough description of some macro-physical processes in the cosmological evolution is given (Sections 14). A possible role of TSL for dark matter is presented (Section 15).

Following a model-driven approach-a methodology widely used in computer science-mathematical formulations are completely omitted from the investigations in this paper. In addition, energy balances are not formulated in the transformations. Many different aspects that can be derived from this new mechanism have to be verified afterwards. We hope to gain the confidence of many other physicists in the YY model. They could extend the core idea of this model in conjunction with quantum field theories and carry out experimental verifications, as well as linking it with existing big bang theories for space and cosmology, especially with the study of dark matter.

\section{Extended Description for up and down Quark}

The starting point is the conventional description of the elementary particles, up quark and down quark, in considering their essential properties: An up quark possesses $2 / 3$ positive of an electrical charge, whereas a down quark has $1 / 3$ negative of an electrical charge. Consequently, two basic building units are elaborated as following:

> "Yin" unit corresponding a third of negative charge, symbolized by "-"

> "Yang" unit corresponding a third of positive charge, symbolized by "+"

These two units, supposed its physical existence, attract each other, and they keep from each other too, so that there is a stable state by "pairing" of them, building a space link, thus called by "pairing space link PSL", as described in Figure 1.

This construction can be expressed by $\{-\mid+\}$. As will be seen later, a space link by pairing is a part of the basic concept of YY model to materialize the energy-matter-space interactions assigned to the pairing. The curly bracket " \{\} " and the pipe sign "|" symbolize this fact.

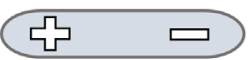

Figure 1. Yin-Yang paring for building a physical space link. 
By using Yin, Yang and PSL, quarks will be described as following:

A down quark contains and materializes a yin-unit, expressed as symbol (-)

An up quark contains and materializes two yang-units and a space link, expressed by $(++\{+\mid-\})$

Drawing the geometric forms of up quark and down quark results in Figure 2. Parenthesis "( )" in the expressions and shadows in the Figures symbolize the other form of the energy-matter-space interactions by the quarks.

\section{Extended Description for Neutron and Proton}

Since a neutron consists of two down and one up quarks and a proton consists of one down and two up quarks (Ref. [2]), the YY model results in the description of neutron and proton in Figure 3.

This re-modeling of neutron and proton reveals more details of their inner structure. The net summation of the positive and negative electrical charges, respectively for a proton and for a neutron corresponds to the classic description. The only difference comes from the space links: they hold the nucleus together.

The following expressions for neutron and proton are equivalent descriptions in Figure 3:

- Neutron: $(++\{+\mid-\})(-)(-)$

- Proton: $(++\{+\mid-\})(-)(\{-\mid+\}++)$

The meta model for the atomic nucleus presented here could lead to concrete predictions for the interaction behavior of neutrons and protons, with each other or with electric fields. For example (here only as a purely theoretical consideration):

$\checkmark$ A neutron has a space orientation respective to the inner charge distribution, namely the axis from the positive to negative pool, as showed in Figure 4(a), though the whole system is electrically neutral. From this point of view, a neutron, released in an electrical field, should orient its positive end towards the negative side of the electrical field, Figure 5(a).

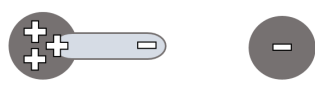

Figure 2. Geometric forms of up quark and down quark.
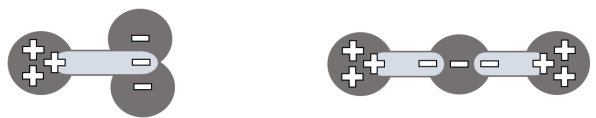

Figure 3. Description model for neutron and proton.

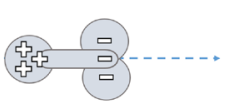

(a)

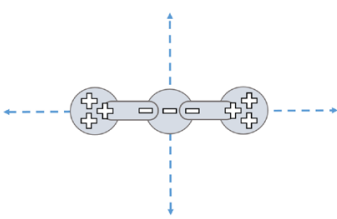

(b)

Figure 4. Rotation axes of neutron and proton. 


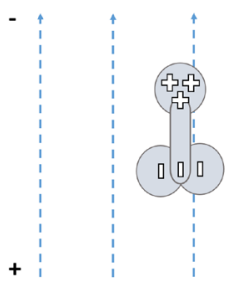

(a)

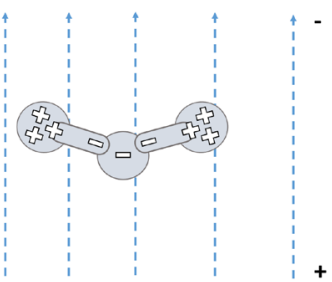

(b)

Figure 5. Orientation of neutron and proton in an electrical field.

$\checkmark$ A proton has a rotation symmetry respective to the axis through the charged poles "positive - negative - positive"; see Figure 4(b). The axis orientation from left to right does not differ from right to left. From this point of view, a proton, released in an electrical field, should tend to orient its symmetry axis perpendicularly to the electrical field, Figure 5(b). A possible electrostatic deformation of the proton structure is also indicated there.

Of course, more research is needed to prove the significance of the effects for an observability, for example whether they can be measured macroscopically as polarization.

By given the proton model, as described above, further descriptions for atomic kernels of the hydrogen isotopes deuterium H-2 and tritium H-3 (Ref. [11]) will continue.

\section{Triple Space Link and Its Role for the Atomic Kernel}

To model complex kernels, a new construct "triple space link (TSL)" is necessary:

> Three PSLs can bind themselves together on their yang units. Their common node corresponds a positive electrical charge unit ("triple charging"), see Figure 6(a);

Similarly, three PSLs can bind themselves together on their yin units to build a negative electrical charge unit; see Figure 6(b).

It is still unclear, how three basic units of a common sign are linked in a node for charging from a physical perspective of view. Furthermore, triple charging is supposed to make an essential difference between the positive and negative nodes in the intensity of binding, as indicated in Figures (a) and (b). The binding on the positive node is strong, whereas the connectivity on the negative node is weak (displayed as gaps) and will be re-connected during the nuclear transformations:

There is an asymmetry between positive charged TSL and negative charged TSL: Whereas a positive charged TSL is pre-created and bound strongly, a negative charged TSL is a mixture of yin pools from positive charged TSLs, of up quarks or of a down quarks. Figure 7 gives all possible combinations for negative charged TSLs, inclusive neutron and proton.

This asymmetry corresponds to the asymmetry between down and up quarks, which will be considered again in a very later section concerning the origins of 


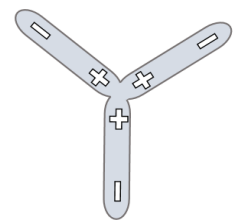

(a)

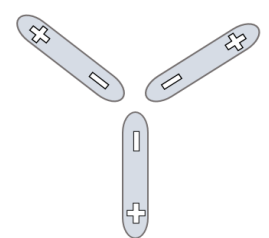

(b)

Figure 6. Triple space links, positive and negative charged.
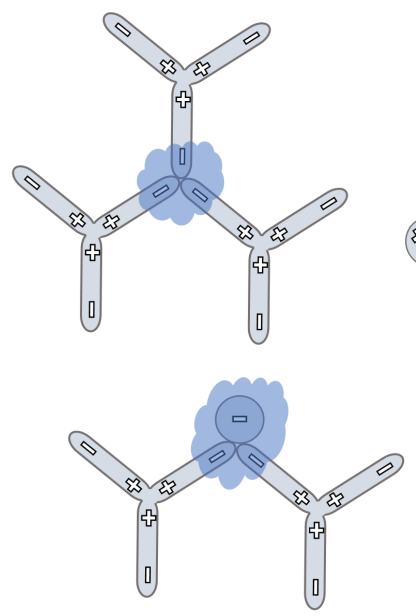
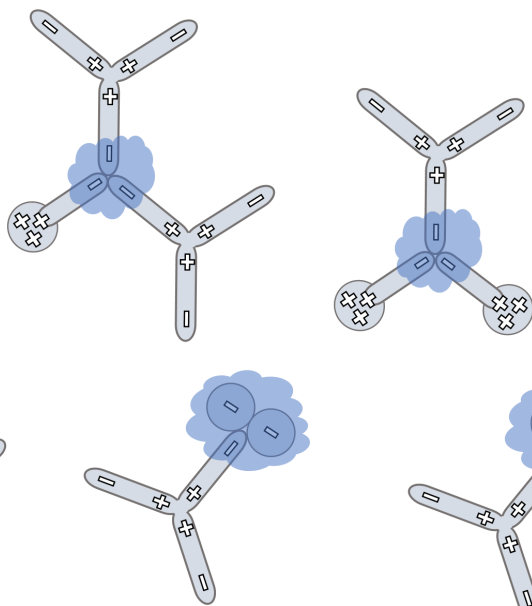
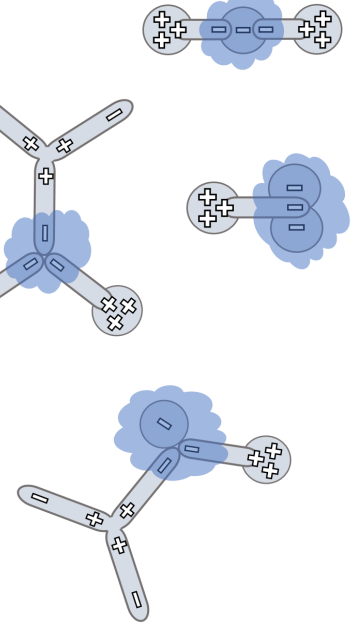

Figure 7. All possible combinations for negative charged TSLs.

cosmology. Due to the asymmetry, speaking of TSL in all following sections we mean exclusively the instances with a positive central node, as drawn in Figure 6(a). Following rules will apply:

$>$ Pairing space link (PSL) can be embedded in an up quark, as described in Section 2;

$>$ Triple pairing space links can be merged on the common positive ends (yang-ends) to build a triple space link (TSL), Figure 6 and Figure 7;

Atomic kernel building is a combination of up quarks by their yin-ends, TSLs by their yin-ends, and down quarks by themselves. Yang-ends stay untouched.

Conservation of Yin's and Yang's: In all transformation processes, the whole number of Yin's and the whole number of Yang's remain unchanged.

\section{Hydrogen Atomic Kernel H-2 and Re-Interpretation for Strong Force}

The starting point is formally going to disassemble an existing proton and an existing neutron and get all their building quarks, see Figure 8. Parts are colored for easy identifying before and after the transformation.

The next step is to reassemble them to a single atomic kernel for building a deuterium (Ref. [11]), with a positive electrical charge unit and a mass unit of two (namely the mass summation of a proton and of a neutron). There are many 


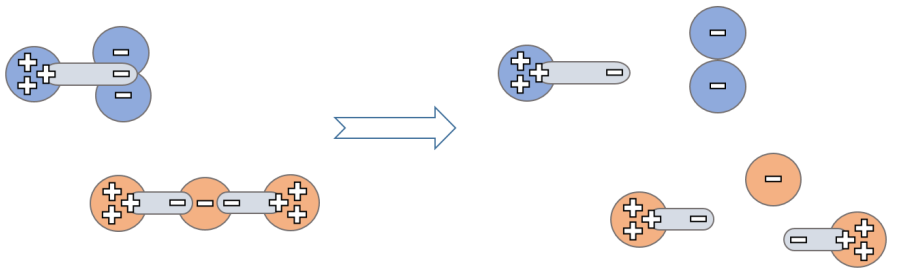

Figure 8. Disassembling a proton and a neutron to their building quarks.

possible ways for reassembling the given quarks, turning out different (quark-) configurations. Only certain configurations of down and up quarks are able to satisfy the charge and mass restrictions for a deuterium.

Starting from rules for quark configurations (at the end of the last section), more rules will be introduced in later sections, while considered atomic kernels become more complex. In the case for reassembling a deuterium, a new triple space link TSL is needed, which combines all given down and up quarks for getting a consistent deuterium atomic kernel, as described in Figure 9.

The upcoming of a TSL in the process of reassembling is a very important point and will be treated in further discussions later.

In consideration of the electrical charges of the whole aggregate above: The net summation of the positive electrical charges is three time $2 / 3$ (=> two positive charge units) and the net summation of the negative electrical charges is three time $1 / 3$ (=> one negative). The net balance of all electrical charges turns out one positive.

In consideration of the mass of the whole aggregate above: The net summation of all three up and all three down quarks results a complete mass of two atomic units, namely three times a third from up quarks, plus three time a third from down-quarks $(3 \times\{1 / 3\}+3 \times\{1 / 3\}=6 / 3=2)$.

The one positive triple link and one negative triple link in the interior of the aggregate are equilibrated in charges.

The strong forces of standard model holding the proton and the neutron together are re-interpreted: The interior negative and positive triple charges of space links hold the surrounding charged up and down quarks together-the holding forces are short-ranged electrostatic forces themselves. QCD based on dynamic color charges of quarks, anti-quarks and gluons (Ref. [12]) is not necessarily used here.

Please also note the drawing of the atomic aggregate in a two-dimensional plane: For a three-dimensional description in the real world, electrostatic attraction and repulsion of up and down quarks around the entire aggregate must be taken into account. In the case of Figure 9, the double down quarks at the top (or/and the double up quarks on the left) must be arranged at least perpendicular to the plane to compensate for electrostatic forces.

\section{Hydrogen Atomic Kernel H-3}

By applying the reassembling schema used above, it is easy to get a consistent 
atomic kernel model for the tritium (Ref. [11]). Firstly, an existing deuterium and an additional neutron are disassembled in Figure 10.

Thereafter, all parts are reassembled together, by adding a new triple space link TSL, resulted as a tritium kernel in Figure 11(a). It is also possible to get a reassembled alternatively model in Figure 11(b).

Please also note the upcoming of a TSL in the process of reassembling.

In consideration of the electrical charges in the aggregate above: The net summation of the positive electrical charges is $4 \times\{2 / 3\}$ (four up quarks times $2 / 3$ ) and the net summation of the negative electrical charges is $5 \times\{1 / 3\}$ (five down quarks times $1 / 3)$. The net balance of all electrical charges turns out one positive $(4 \times\{2 / 3\}-5 \times\{1 / 3\}=3 / 3=1)$.

In consideration of the mass in the aggregate above: The net summation of all four up and all five down quarks results a complete mass of three atomic units (four times a third from up quarks, plus five time a third from down-quarks $4 \times$ $\{1 / 3\}+5 \times\{1 / 3\}=9 / 3=3)$.

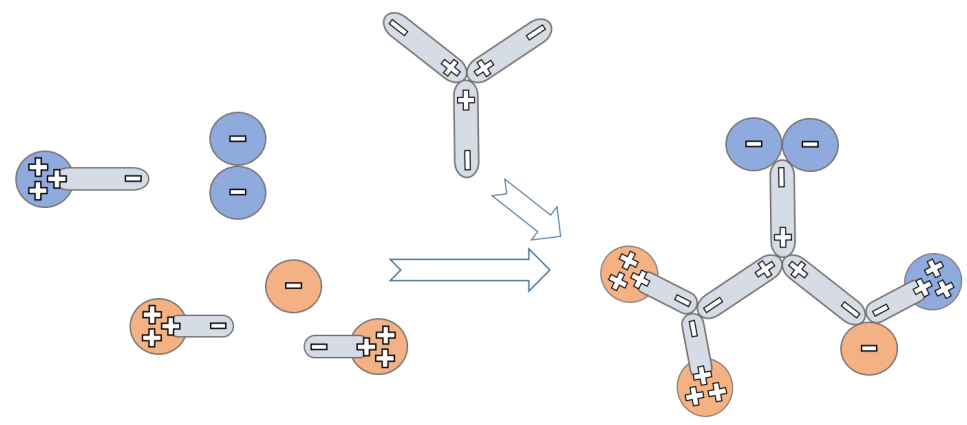

Figure 9. Reassembling the given quarks to a deuterium by adding a TSL.
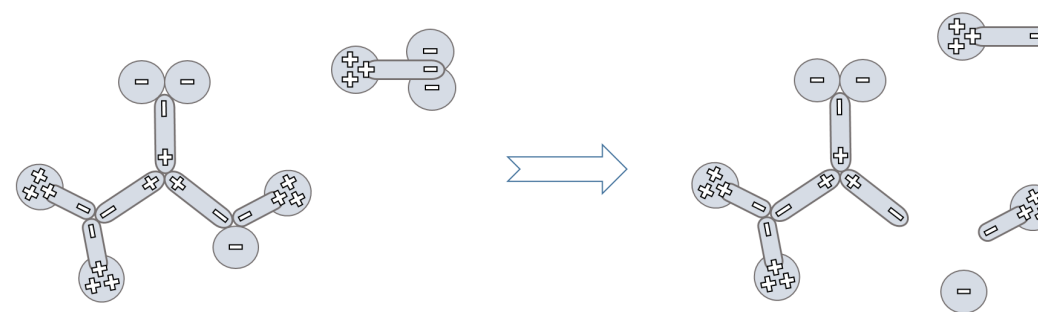

Figure 10. Disassembling a deuterium and a neutron.

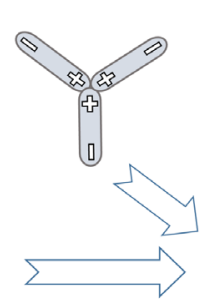

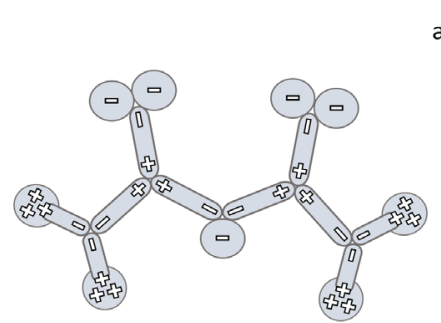

(a)

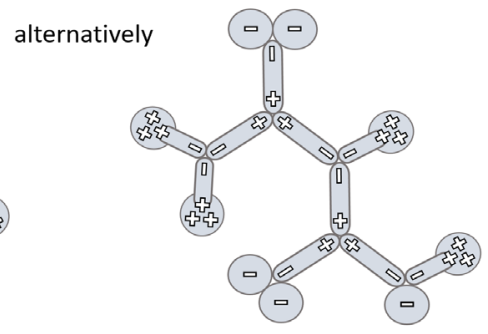

(b)

Figure 11. Two alternative tritium kernel models reassembled by adding a TSL. 
The two positive triple nodes and two negative triple nodes in the interior of the aggregate are equilibrated in charges.

Similarly, the classic strong forces holding the proton and the neutrons are re-interpreted by links of different sorts. This is the same mechanism for holding one proton and one neutron together.

\section{Helium Kernel He-4 and Its Isotope He-3}

This section will describe the helium atomic kernel He-4 in YY model, constructed by two protons and two neutrons. Furthermore, one of its isotopes He-3 will also be considered, with two protons plus one neutron.

In the case of $\mathrm{He}-4$, a formal reassembling from two $\mathrm{H}-2$ atomic kernels is done. Figure 12 firstly disassembles two H-2 atom kernels respectively.

By adding one triple space link TSL and reassembling them all, a stable helium kernel He-4 is resulted, see Figure 13.
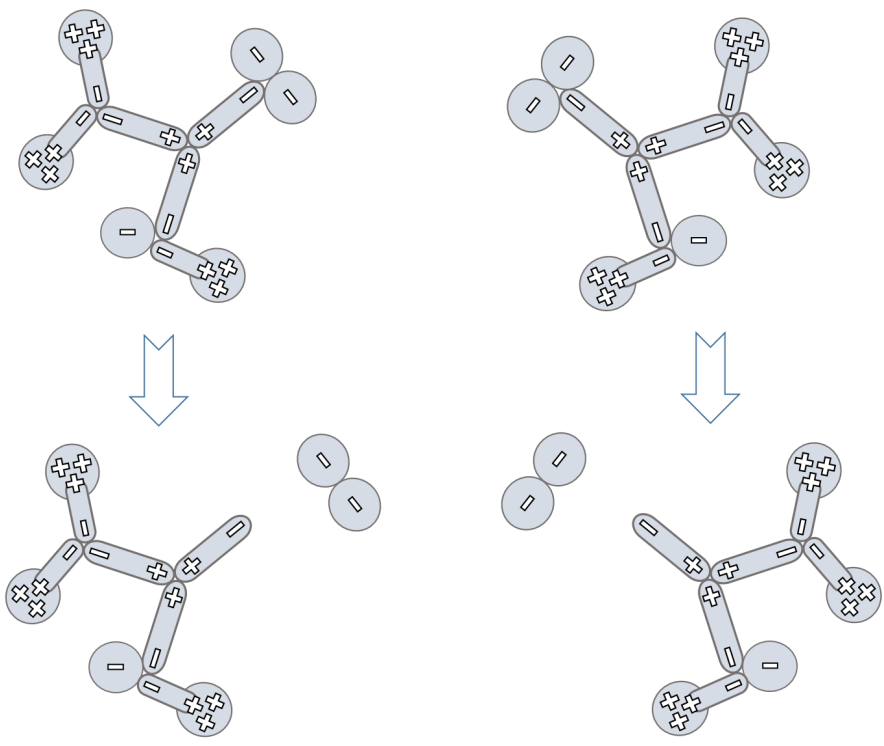

Figure 12. Disassembling two hydrogen atomic kernels H-2.
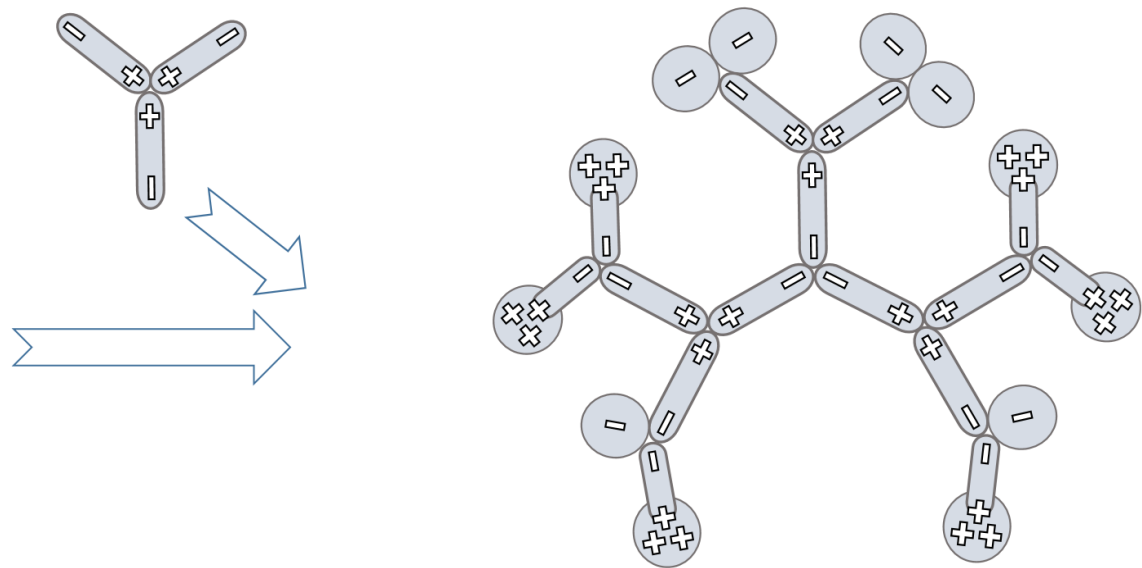

Figure 13. Reassembled atomic kernel for a helium- 4 from Figure 12 by adding a TSL. 
Consideration of the electrical charges: The net summation of the positive electrical charges is $6 \times\{2 / 3\}$ (six up quarks times 2/3) and the net summation of the negative electrical charges is $6 \times\{1 / 3\}$ (six down quarks times $1 / 3$ ). The net balance of all electrical charges turns out two positive $(6 \times\{2 / 3\}-6 \times\{1 / 3\}=6 / 3$ $=2$ ).

Consideration of the mass: The net summation of all six up and all six down quarks results a complete mass of four atomic units (six times a third from up quarks, plus six time a third from down quarks $6 \times\{1 / 3\}+6 \times\{1 / 3\}=12 / 3=4$ ).

The three positive triple nodes and three negative triple nodes in the interior of the aggregate are equilibrated in charges.

The same mechanism is used for holding up and down quarks together, which is a re-interpretation for the strong forces.

As next step, atomic kernel of the helium isotope He-3 is considered, firstly by disassembling a hydrogen $\mathrm{H}-1$ and a deuterium $\mathrm{H}-2$ kernel respectively, as displayed in Figure 14.

By adding one triple space link TSL and reassembling them all, the result helium kernel He-3 is resulted in Figure 15.

Consideration of the electrical charges: The net summation of the positive electrical charges is $5 \times\{2 / 3\}$ (five up quarks times $2 / 3$ ) and the net summation of the negative electrical charges is $4 \times\{1 / 3\}$ (four down quarks times $1 / 3$ ). The net balance of all electrical charges turns out two positive $(5 \times\{2 / 3\}-4 \times\{1 / 3\}=$ $6 / 3=2)$.
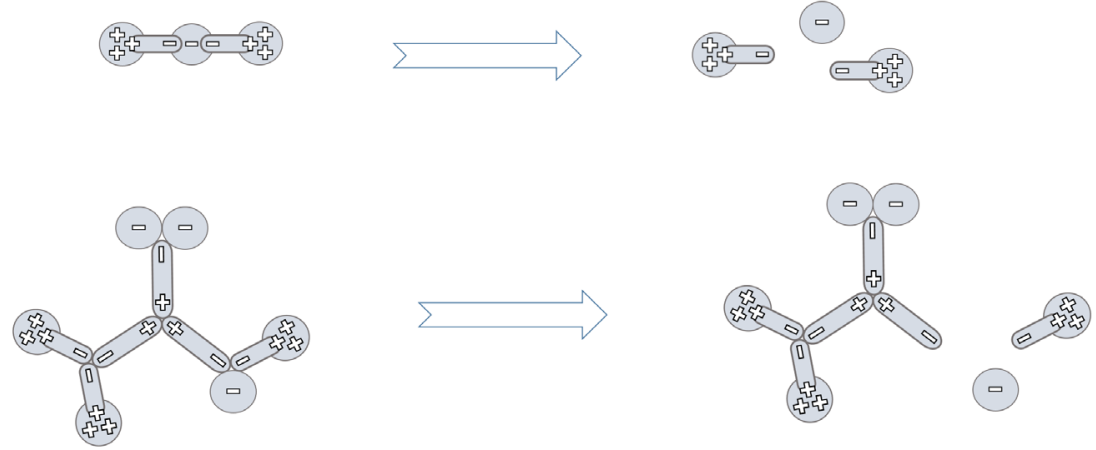

Figure 14. Disassembling hydrogen $\mathrm{H}-1$ and deuterium $\mathrm{H}-2$ kernel.
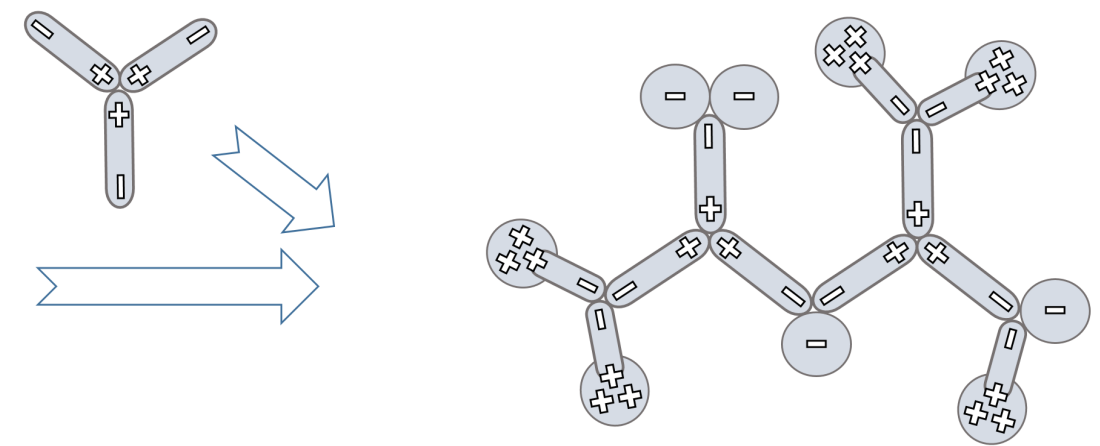

Figure 15. Reassembled atomic kernel for a helium-4 from Figure 12 by adding a TSL. 
Consideration of the mass: The net summation of all five up and all four down-quarks results a complete mass of three atomic units (five times a third from up-quarks, plus four time a third from down-quarks $5 \times\{1 / 3\}+4 \times\{1 / 3\}=$ $9 / 3=3)$.

The two positive triple nodes and two negative triple nodes in the interior of the aggregate are equilibrated in charges.

\section{Alternative Kernel Configurations for Helium He-4}

YY model makes it possible that multiple consistent atomic kernel models (alternative configurations) for helium He-4 exist. One of the alternatives to Figure 13 is to reassemble the parts in Figure 12, by adding two triple space links, to Figure 16.

Another alternative is, also by adding two triple space links, to reassemble the parts from Figure 12-17.

All common things of models in Figure 13, Figure 16 and Figure 17 for helium atomic kernel $\mathrm{He}-4$ are

- The numbers of up quarks (6) and the numbers of down quarks (6);

- The electrical charge balance (positive 2), and

- The mass summation units of all quarks (3).

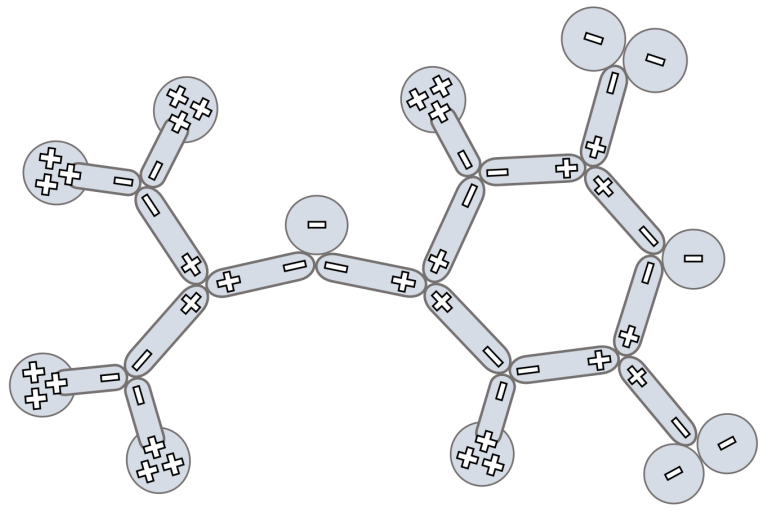

Figure 16. A Possible alternative atomic kernel model for helium He-4.

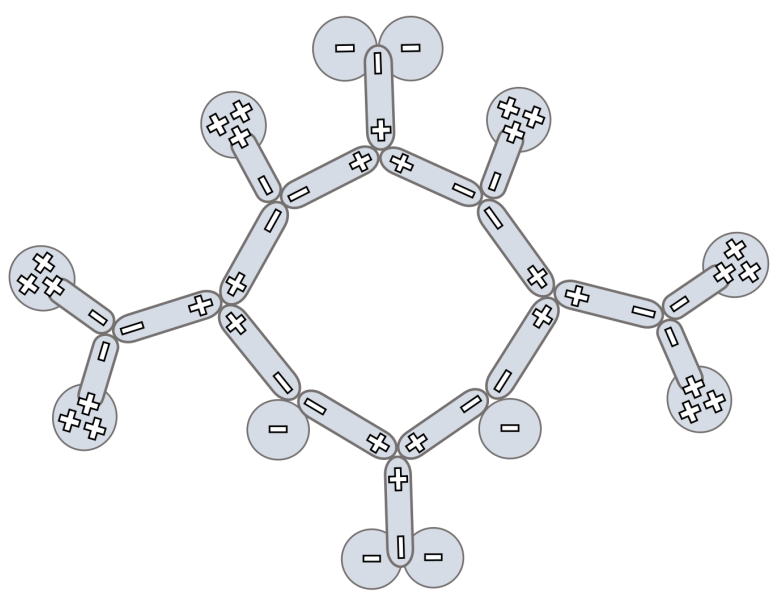

Figure 17. Another possible alternative atomic kernel model for Helium He-4. 
The internal triple space links vary from alternatives: Figure 13 possesses three positive TSLs and their balancing negative nodes; Figure 16 and Figure 17 possess four positive TSLs and four balancing negative nodes respectively. Generally as a configuration rule:

- The number of positive TSLs is equal to the number of negative balancing nodes (Internal Charge Balance ICB).

ICB is important for the stability of aggregate structures, though the governing mechanism must be still investigated mathematically and physically.

Moreover, a TSL must possess a mass unit that is essential smaller in comparing to the mass of an up or down quark-these are in the order of a third atomic mass unit.

\section{Process for Generating Electron and Positron}

In this section, we discuss how the YY model understands the electron and positron, their generation processes using the prototype for the electron and splitting the quarks for the positron. The construction and composition of the electron and positron are found out here.

To generate an electron, the YY model formally considers a nuclear interaction of two neutrons with each other (dis- and reassembly). One proton and three "liberated" down quarks can be obtained, which together form a prototype for a negatively charged particle that decays successively into an electron and neutrino/antineutrino of different generations, as shown in Figure 18.

Essential aspects for generating electron are:

An electron in the YY model is composed of deep-bound yin's, originated from three down quarks (likely a positive triple space link is composed of deep-bound yang's from three paring links);

Kernel transformation model: $2 \mathrm{n} \rightarrow \mathrm{p}^{+}+$electron prototype $\rightarrow \mathrm{p}^{+}+\mathrm{e}^{-}+$neutrino, antineutrino.

The main difference between YY model and the well-known processes in standard model like muon decay $\left(\mu^{-} \rightarrow \mathrm{e}^{-}+\bar{v}_{\mathrm{e}}+v_{\mathrm{u}}\right)$ and beta decay $\left(\mathrm{n} \rightarrow \mathrm{p}^{+}+\mathrm{e}^{-}+\bar{v}_{\mathrm{e}}\right)$ (Ref. [11] and [13]) is the involved number of neutrons. Furthermore, the electron origination (prototype) and resulted properties (deep-bound three yin's) differ.

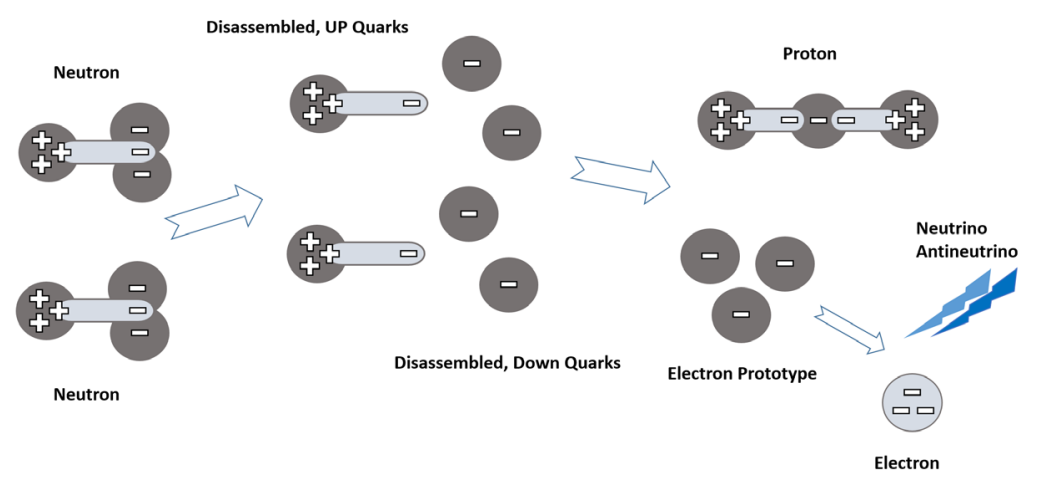

Figure 18. Generating process for electron. 
Generating positron is interpreted by the "inverse beta decay" (Ref. [11]): $\overline{\mathrm{v}}_{\mathrm{e}}+\mathrm{p} \rightarrow \mathrm{e}^{+}+\mathrm{n}$, as displayed in Figure 19.

Obviously, the antineutrino strikes one up quark, shifted the mass (grey shadowed) from its positive charge pool to its negative pool becoming a down quark to combine the rest of the aggregate to a neutron, while the positive part of the concerned up quark becomes a positron.

Of course, the widely accepted standard particle model postulates that a free electron does not possess a measurable dimension and, that an electron is not dividable to smaller elementary particles.

From the perspective of YY model, electron as deep-bound yin's can still have a smaller dimension unit than our current physical detective ability. Furthermore, electron as "no dividable particle" is also a fact, with one difference:

The only way to reassemble an electron is its collision with a positron that leads to annihilation of the both particles and emission of gamma radiations. In the YY model, this collision will create a reassembled triple space link TSL (see next section) which is "given back" to the space and becomes a "waste" matter.

\section{Electron-Positron Annihilation in YY Model, Outcome TSL and Y Particle}

The YY model conserves the participating yin's and yang's in all transformation processes. The electron-positron annihilation described in standard model (Ref. [11] [14] $) \mathrm{e}^{+}+\mathrm{e}^{-} \rightarrow 2 \gamma$ will be extended in YY model by generating a TSL obeying this conservation, see Figure 20.

TSL is a prediction of the YY model. It can be imagined as ashes after the wood has burned out. Each TSL has a mass defined as a "TSL mass unit", which must be much smaller than the mass of electron and positron, but still not

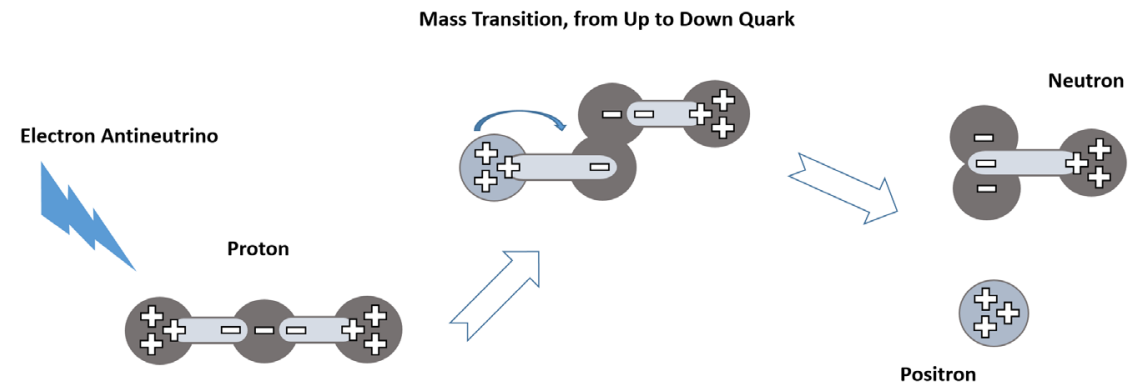

Figure 19. Generating process for positron.

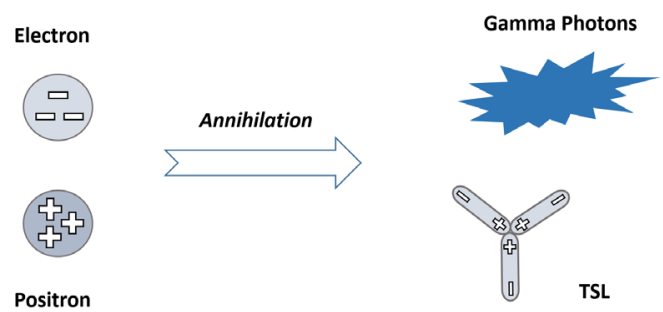

Figure 20. Electron-positron annihilation by generating gamma photons and a TSL. 
negligible. It might be possible to determine it indirectly, using a more precise energy-matter balance between predictions and observations, also to estimate the absolute value of the TSL unit of mass.

Actually, electron-positron annihilation is part of the energy-matter life cycle and TSLs go back to their original form, which is given at the beginning of cosmological evolution. The third to last section will give a rough description of this life cycle. In addition, the TSL formation forms the basis for the antimatter systematics of the YY model (outside the scope of this paper).

We call TSL as Y particle, in order to motivate physicians to verify its existence.

\section{Rules for Electrical Charge Units and Mass Units of Atomic Kernel}

The early modelling approaches in this paper have showed, that the net electrical charges of an atomic kernel turn out from the summation of all building outer vertexes - all up and down quarks combinations. For clearness, some more terminologies are introduced here (see also Figure 21).

- Neutronhead: Triple end node containing two down quarks-possesses one negative electrical charge unit and 2/3 atomic mass unit;

- Protonhead: Triple end node containing one down quark-possesses one negative electrical charge unit and $1 / 3$ atomic mass unit;

- Protonid: Single end node containing one up quark-possesses one positive electrical charge unit and 1/3 atomic massunit.

Additionally to the properties for electrical charges and mass units described above, there are also rules for balancing the numbers of protons and neutrons in an atomic kernel:

- Every protonhead needs two corresponding protonids for building a proton within an atomic kernel aggregate (number of protonheads $=$ number of protons);

- Every neutronhead needs one corresponding protonid for building a neutron within an atomic kernel aggregate (number of neutronheads = number of neutrons).

Thus, the occurrences of protonids within an atomic kernel aggregate are not determining factor for calculating the numbers of protons and neutrons: They are only for satisfying the other requirements from the occurrences of protonheads and neutronheads.

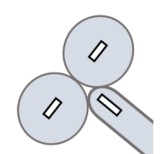

Neutronhead

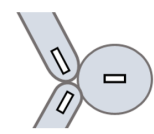

Protonhead

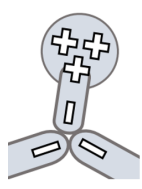

Protonid

Figure 21. Neutronhead, protonhead and protonid. 
This implies another asymmetry between up quark and down quark: The occurrences of down quarks (single or in pair) are essential for determining the protons and neutrons assembled in an atomic kernel.

Furthermore, a special rule has to be obeyed by the whole aggregate:

- The number of positive triple space links (TSLs) and the number of negative binding vertexes must balance in total (=> Internal Charge Balance ICB), as already discussed in the early Figure 9, Figure 11, Figure 13, Figure 16 and Figure 17.

In Figure 22 for the He- 4 atomic kernel, colored texts annotate the internal charge balance (blue), respectively the external charge balance (red). "Internal" means pairing space links (PSLs) embedded within triple space links (TSLs). "External" means all surrounding protonheads, neutronheads and protonids.

The internal charge balance (ICB) guarantees the electrical neutrality of the binding element structure (PSLs, TSLs) so that the whole electrical charge balance of an atomic kernel just results from the summation of all surrounding protonheads, neutronheads and protonids.

All these rules give us the guidelines for assembling and reassembling new atomic kernels by disassembling the original atomic kernels, in a consistent way.

The atomic mass unit calculation for a kernel aggregate is simple and straightforward:

- The atomic mass units of an atomic kernel results from the summation of all atomic mass units of surrounding protonheads, neutronheads and protonids, divided by three;

- The TSL mass units results from the number of existing TSLs.

In Figure 23 for the He-4 atomic kernel, colored texts annotate atomic mass units of all protonheads, neutronheads and protonids (green), respectively the TSL mass units (blue).

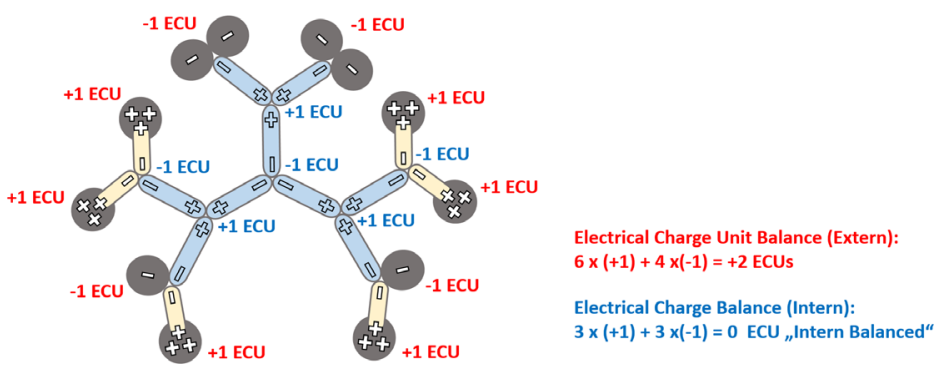

Figure 22. Electrical charge balances of helium kernel He-4.

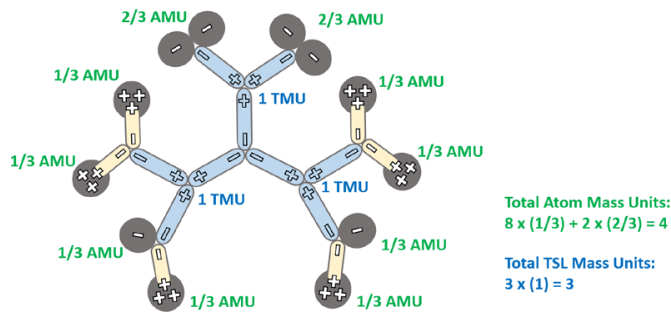

Figure 23. Atomic mass units and TSL mass units of helium kernel He-4. 


\section{Stellar Nuclear Fusion Process}

This section describes some important parts of the stellar fusion reactions (Ref. [15] and [16]) - divided into four sub-sections. The mechanisms of the YY model discussed in the early sections are applied here to understand the internal structural changes in the fusion reactions without considering details of the energy transitions.

\subsection{Nuclear Fusion to Deuterium $\mathrm{H}-2$}

The beginning stage of stellar nuclear fusion produces a hydrogen isotope $\mathrm{H}-2$ by merging two protons $\mathrm{H}-1$ and generating a positron and neutrino:

$\mathrm{H}-1+\mathrm{H}-1 \rightarrow \mathrm{H}-2+\mathrm{e}^{+}+v$.

Following the same construction procedure by disassembling the both $\mathrm{H}-1$ and reassembling their elements to get $\mathrm{H}-2$ get applied here. To ensure the model consistence by obeying the rules (last section), a new triple space link TSL is needed (Figure 24).

The complete transformation can be seen in two steps. In the first step, a proton is virtually transformed into a neutron by an inverse beta decay (Figure 19) and, in a further step, the virtual neutron and a second proton are merges into a deuterium (Figure 9 ) by adding a TSL.

The interpretation of the first step is equivalent to assuming the transition of an up quark to a down quark, a positron and an electron neutrino $\left(\mathrm{u} \rightarrow \mathrm{d}+\mathrm{e}^{+}+v_{\mathrm{e}}\right)$, Figure 25.

However, what happens to the excessive positron? Moreover, where does TSL come from? Obviously, the both questions have something strongly to do with
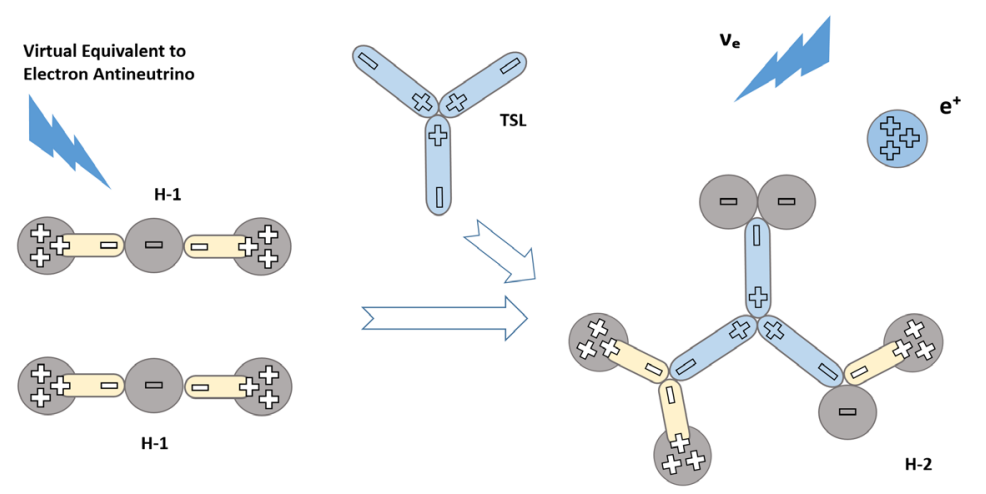

Figure 24. Nuclear fusion to H-2.

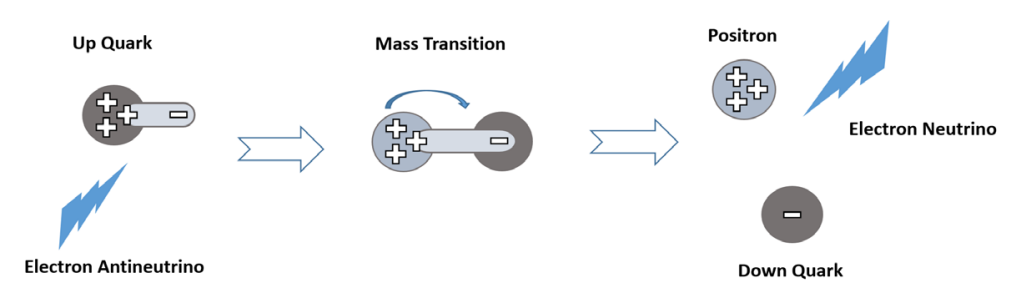

Figure 25. Transition of an up quark to down quark, positron and neutrino. 


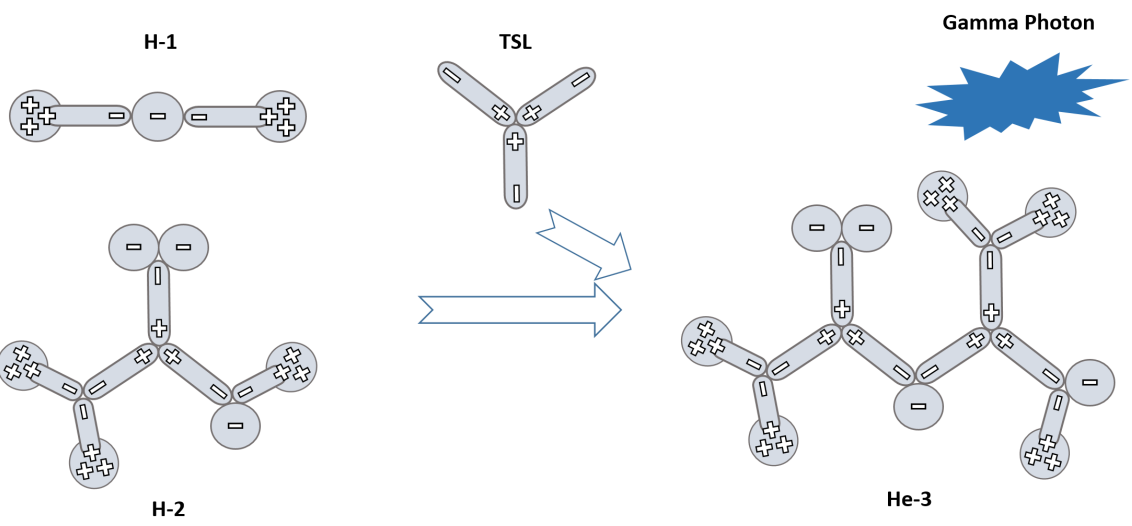

Figure 26. Nuclear fusion to He-3.

each other. This will be explained in the Section 12.4, "Mechanism for Producing TSLs in Stellar Nuclear Fusion".

\subsection{Nuclear Fusion to Helium Isotope $\mathrm{He}-3$}

The next stage of stellar nuclear fusion produces a helium isotope He-3 by merging a proton $\mathrm{H}-1$ and a hydrogen isotope $\mathrm{H}-2$, emerging gamma photon: $\mathrm{H}-1+\mathrm{H}-2 \rightarrow \mathrm{He}-3+\gamma$ (Figure 26). Following the YY model, a new TSL is needed, accompanying the gamma emerging.

\subsection{Nuclear Fusion to Helium He-4}

In a further stage of stellar nuclear fusion, a stable helium He-4 and two protons $\mathrm{H}-1$ will be produced by merging two helium isotopes $\mathrm{He}-3$ :

$\mathrm{He}-3+\mathrm{He}-3 \rightarrow \mathrm{He}-4+2 \mathrm{p}+12.9 \mathrm{MeV}$.

The structure of helium He-3 is already described in early section (Figure 15). The fusion process after YY model is described in Figure 27. To illustrate clearly, and for better identifying, parts are colored in aggregates before and after the fusion.

Be aware that this fusion stage releases an existing TSL, which can be re-consumed in the both of early fusion stages for $\mathrm{H}-2$ and He-3.

\subsection{Mechanism for Producing TSLs in Stellar Nuclear Fusion}

Two stellar nuclear fusion stages consume TSLs, as already described in Section 12.1 and 12.2 (Figure 24 and Figure 26). This section explains the mechanism for producing TSLs: It comes from two sources:

- Released from the fusion stage to build He-4 (see Figure 27), and

- Generated from the beginning fusion stage to build H-2-this will be described in following.

The generation mechanism is composed of two nuclear transformation parts inside the fusion process $\mathrm{H}-1+\mathrm{H}-1 \rightarrow \mathrm{H}-2+\mathrm{e}^{+}+v$ :

$\checkmark$ Virtual inverse beta decay (Figure 19): $\overline{\mathrm{e}_{\mathrm{e}}}+\mathrm{p} \rightarrow \mathrm{e}^{+}+\mathrm{n}$

$\checkmark$ Electron-proton annihilation (Figure 20): $\mathrm{e}^{+}+\mathrm{e}^{-} \rightarrow 2 \gamma+\mathrm{TSL}$ 

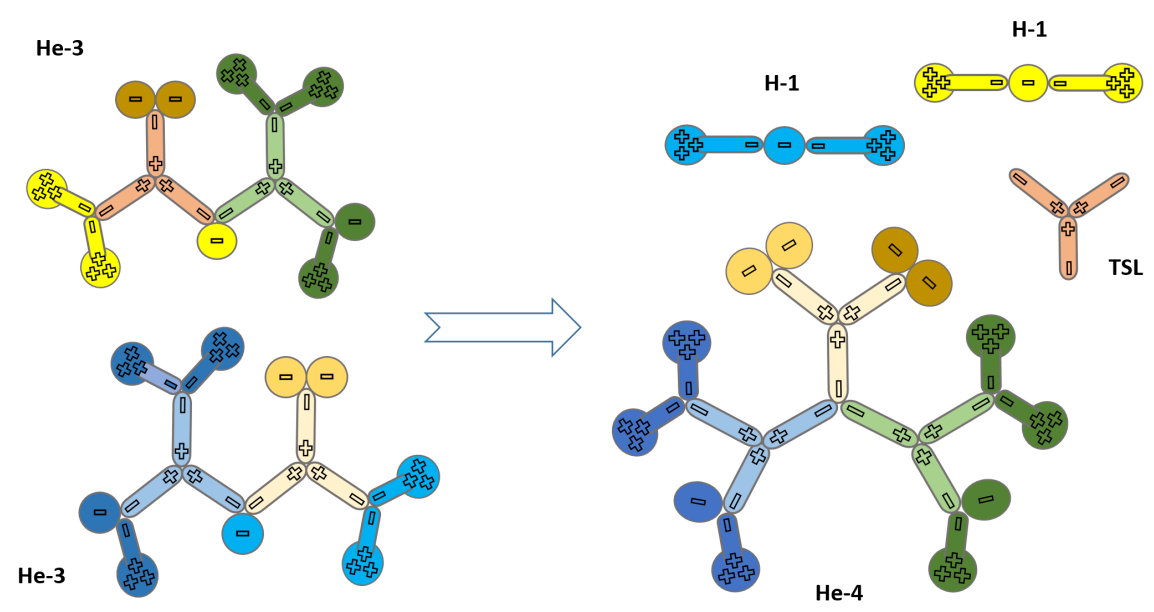

Figure 27. Nuclear fusion to He-4.

What happens is only possible to consider a pair of hydrogen atoms (two hydrogen kernels and two electrons) in a high-density state (Ref. [16]):

$$
\begin{aligned}
& 2 \mathrm{H}-1+2 \mathrm{e}^{-} \text {, ionized \& energized } \\
& \rightarrow \overline{v_{\mathrm{e}}}+\left(\mathrm{p}^{+}+\mathrm{e}^{-}\right)+\left(\mathrm{p}^{+}+\mathrm{e}^{-}\right) \rightarrow\left(\mathrm{e}^{+}+\mathrm{n}+\mathrm{e}^{-}\right)+\left(\mathrm{p}^{+}+\mathrm{e}^{-}\right) \\
& \rightarrow(2 \gamma+\mathrm{TSL}+\mathrm{n})+\left(\mathrm{p}^{+}+\mathrm{e}^{-}\right) \rightarrow 2 \gamma+\left(\mathrm{TSL}+\mathrm{n}+\mathrm{p}^{+}\right)+\mathrm{e}^{-} \\
& \rightarrow 2 \gamma+\mathrm{H}-2+\mathrm{e}^{-}
\end{aligned}
$$

With the considered group of two hydrogen atoms (already ionized), one up quark of the first $\mathrm{H}-1$ is transformed to a neutron, producing one positron which is soon annihilated together with the first electron, producing gamma photons and a TSL. By consuming this TSL, the second H-1 merges with the transformed neutron to a deuterium kernel H-2 (Figure 9). The second electron balances this merged kernel electrically. The standard description $\left(\mathrm{H}-1+\mathrm{H}-1 \rightarrow \mathrm{H}-2+\mathrm{e}^{+}+v\right)$ gets here a more precise and detailed formulation.

The self-providing mechanism for TSL will also take effect for the second stage (Figure 26): $\mathrm{H}-1+\mathrm{H}-2 \rightarrow \mathrm{He}-3+\gamma$, with the following expression concerning the participating hydrogen atom $\mathrm{H}-1$ :

$$
\bar{v}_{\mathrm{e}}+\left(\mathrm{p}^{+}+\mathrm{e}^{-}\right)+\cdots \rightarrow\left(\mathrm{e}^{+}+\mathrm{n}+\mathrm{e}^{-}\right)+\cdots \rightarrow(2 \gamma+\mathrm{TSL}+\mathrm{n})+\cdots
$$

Lastly, the third stage of fusion process for building stable helium He-4 releases a TSL, which can participate other fusion stages.

TSL emergency and its involvement in the nuclear fusion processes is new and important in comparison to the standard model. TSL is not easily detectable, thus out of focus of our experimental physics until now. It is a stable state of matter occurrence.

\section{Essentials of a Nuclear Fission Process}

A very essential thing happened in association with a nuclear fission process will be considered in this section: How a separation occurs at the fraction place, based on the YY model.

Consider the example nuclear fission of uranium atoms (Ref. [11]): 


$$
\mathrm{n}+\mathrm{U}-92 / 235 \rightarrow \mathrm{Ba}-56 / 139+\mathrm{Kr}-36 / 95+2 \mathrm{n}+200 \mathrm{MeV}
$$

Without modelling the complete big atomic kernel of uranium 235 (that would massively extend the scope of this paper), the single place is interested which separates the uranium atom kernel into the both parts of $\mathrm{Ba}-139$ and Kr-95.

The YY model will describe this place with a possible structure configuration before and after separation in Figure 28. Colored parts help to identify them in the structure of big kernel before and kernel pieces after the separation.

Not only up and down quarks are preserved, but the electrical charges before and after the separation. Additionally, two existing triple space links TSLs are released.

Allowedly, the model description above supposes, that there is a single place to separate the U-235 atom kernel to its fission products $\mathrm{Ba}-139$ and $\mathrm{Kr}-95$. It is possible the separation occurs cross multiple connected places - the final answers will turn out after modelling the complete uranium atoms. Many different valid structure configurations are possible, as the simple cases for tritium $\mathrm{H}-3$ (Figure 11), and for helium He-4 (Figure 13, Figure 16 and Figure 17) already illustrated.

Cold Fusion:

The YY model provides additional arguments that can help to understand whether cold fusion (Ref. [17]) can work.

A nuclear reaction without the consumption of TSL can be considered as "cold" because no electron-positron annihilation takes place and therefore no gamma radiation is produced. Transformation processes that even release TSLs can offer them for parallel running fusions, which in turn consume TSLs without producing them themselves.

Stellar fusion processes that require TSLs for binding, as already described for the formation of $\mathrm{H}-2$ and He-3, must themselves "produce" TSLs and thus gamma photons.

Because the nuclear fission $(n+U-92 / 235 \rightarrow$ Ba-56/139 + Kr-36/95 $+2 n+\cdots)$
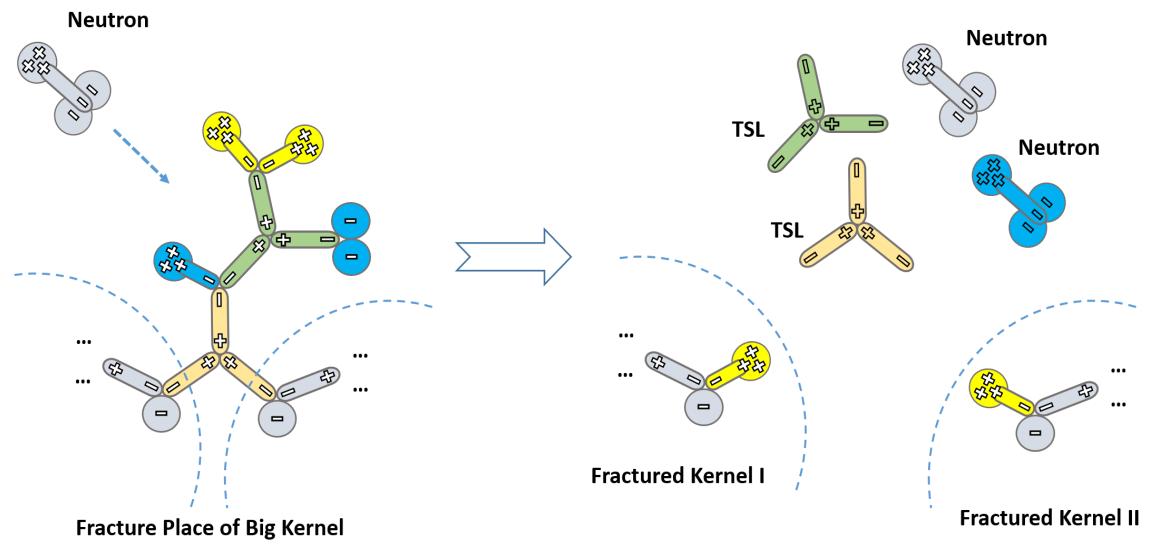

Figure 28. Fracturation of a big atomic kernel and its products. 
also releases TSLs, it is possible that other big atomic kernels (not necessarily uranium) also do this so that these TSLs can be consumed by parallel running fusions without (or with few) gamma radiations.

Figure 29 is the YY model description for well-known nuclear fusion process for helium He-4 (Ref. [16]): $\mathrm{H}-2+\mathrm{H}-3 \rightarrow \mathrm{He}-4+3.5 \mathrm{MeV}+(n+14.1 \mathrm{MeV})$.

No TSL is involved in this process, neither consumed nor released. Gamma radiations $\left(\mathrm{e}^{-}+\mathrm{e}^{+} \rightarrow \mathrm{TSL}+2 \gamma\right)$ do not occur. Thus, hydrogen fusion from $\mathrm{H}-2$ and $\mathrm{H}-3$ to helium He-4 becomes a good technical candidate for nuclear cold fusion, being interesting for human applications.

It should be mentioned, that the considerations here are only from the structure point of view. Energy balance aspects are not touched.

\section{A Possible Scenario for Matter Evolution during Cosmological Inflation}

If, as already mentioned, we go back to the origin of TSLs from the beginning of the cosmological evolution, we find some concrete construction processes that are closely related to the inflation phase (Ref. [18]). They are described in the following subsections as life cycle parts of a possible scenario.

\subsection{Triple Space Link and Its Role for the Homogeneous Space}

At an early stage during the inflation phase after the big bang, before the build-up of real matter, space should have a homogeneous structure caused by a high energy density. The YY model considers this homogeneous structure as composed of interconnected TSLs (called "Triple Space Linked State" TSLS). It is the initial state for further matter formation, Figure 30.

It is mainly a spatial structure based on electrostatic interactions of TSLs. The matter-antimatter asymmetry of the cosmos can already be founded here. At this stage of cosmological expansion, space is "cross-linked" by interconnected TSLs - The cosmos is filled with Y-particles.
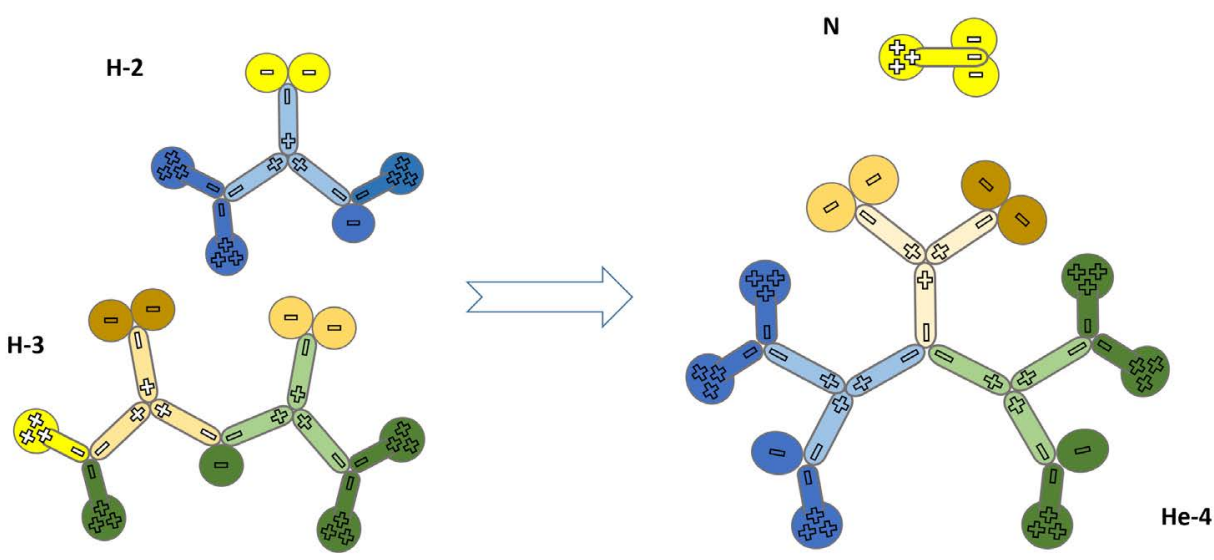

Figure 29. Fusion of deuterium and tritium to helium, accompanied by a neutron. 


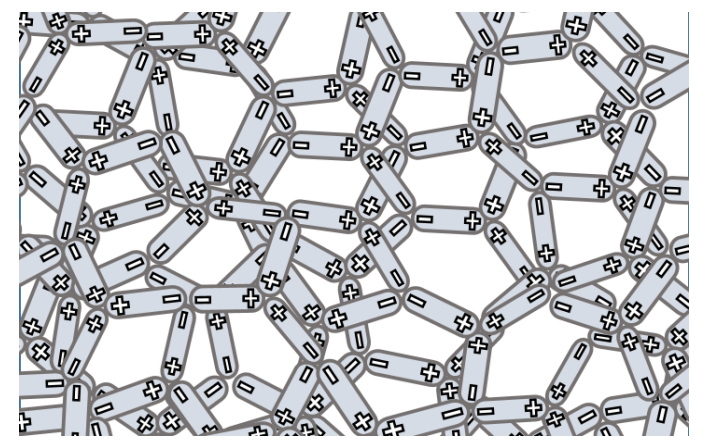

Figure 30. Triple space linked state.

\subsection{Pre-Build Matter in Form of up and down Quarks from TSLS}

In this stage, inter-linked TSLs will be released from each other. Up quarks and down quarks will be created from TSLs as manifestation of energy to matter: Every TSL itself, forced by energy, can be "transformed" into one up quark and two down quarks - cutting two yin's and fill all parts with matter, see Figure 31.

Because up and down quarks are dependent matter form, "pre-build" of matter is used here. At this stage, space is filled with up quarks, twice times numbers more with down quarks and rest TSLs, Figure 32.

\subsection{Build Neutrons}

The following stage builds neutrons, whereas the space is expanding and more transparency for particles and more freedom for pre-build matters given. One neutron is assembled by "tripling" one up and two down quarks, Figure 33.

Building neutrons can be regarded as one scenario. It is also possible that protons are built with existing up and down quarks parallel to neutrons.

\subsection{Build Protons and Electrons}

As already described in Figure 18 of Section 9, pairs of neutrons can reassemble themselves to protons and electrons. The universe filled with neutrons (Figure 33) will than descend soon into a universe filled partially with protons, electrons and rest neutrons (Figure 34):

Neutrinos and antineutrinos are emerged (not drawn in the Figure) by electron prototypes as mediator.

\subsection{Pairing of Protons and Electrons to Hydrogen Atoms H-1}

With expansion of space, free electrons are bound together with protons to form hydrogen atoms (Figure 35). Later hydrogen atoms are grouped to hydrogen molecules by chemical binding.

Two essential details still need to be explored: First, how cosmological inflation leads to a state "filled with" triple space links by taking into account how high energetic interactions take place while space expands at a very early stage of inflation. Secondly, how the energies manifest themselves in the subsequent expansion process to form pre-fabricated matter (up and down quarks). 


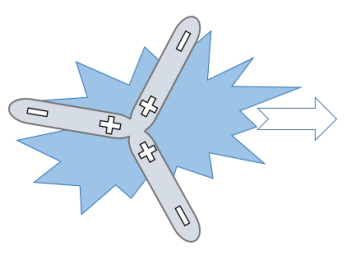

TSL forced by Energy

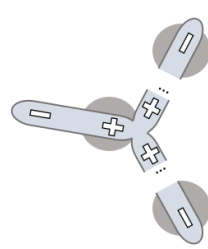

Materialization of Energy

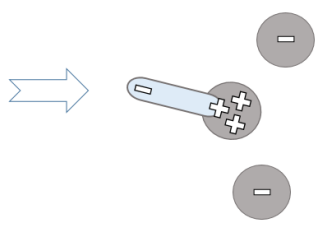

Up and Down Quarks

Figure 31. From one TSL to one up quark and two down quarks.

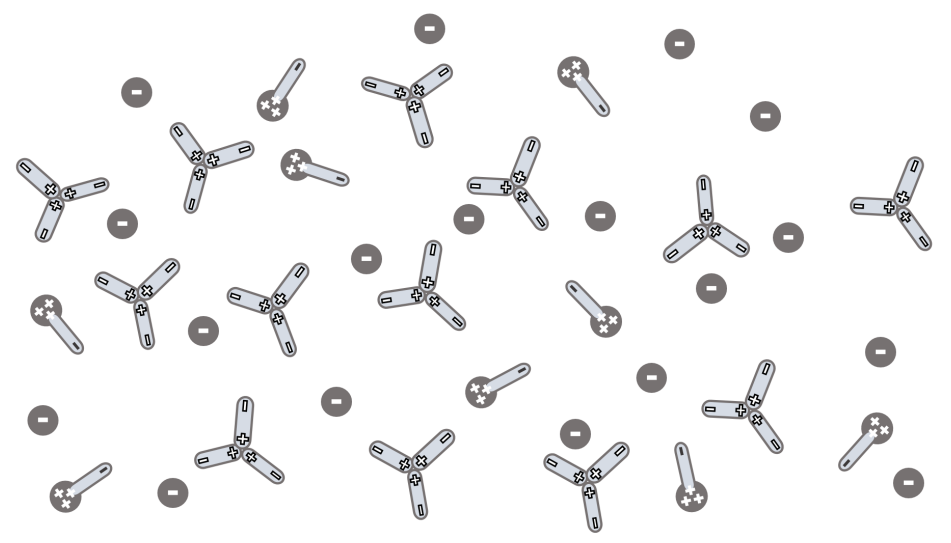

Figure 32. Universe filled with up quarks, down quarks and TSLs.

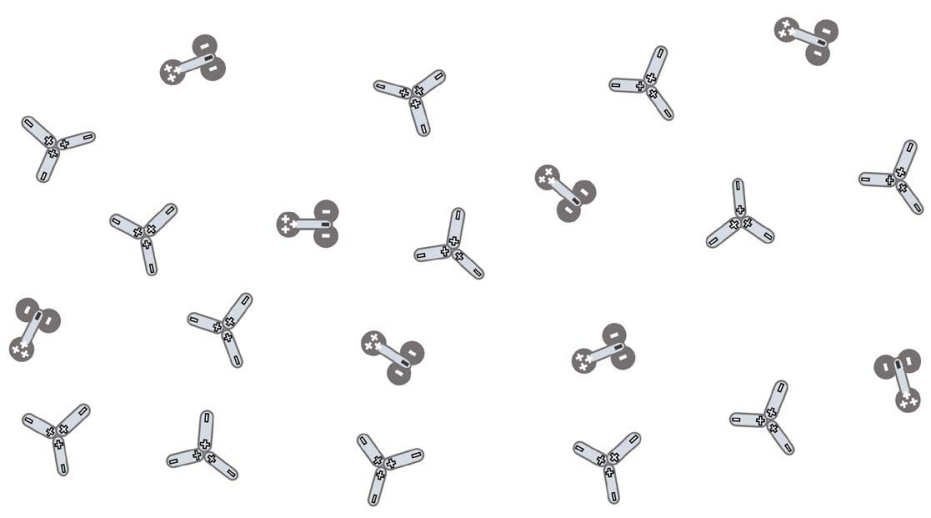

Figure 33. Universe filled with neutrons and TSLs.

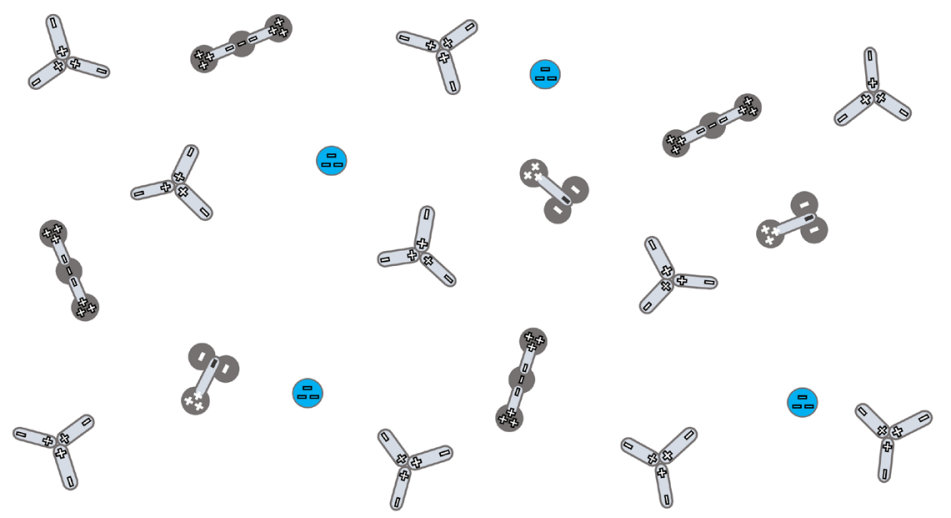

Figure 34. Universe filled with protons, electrons and neutrons, accompanied by TSLs. 


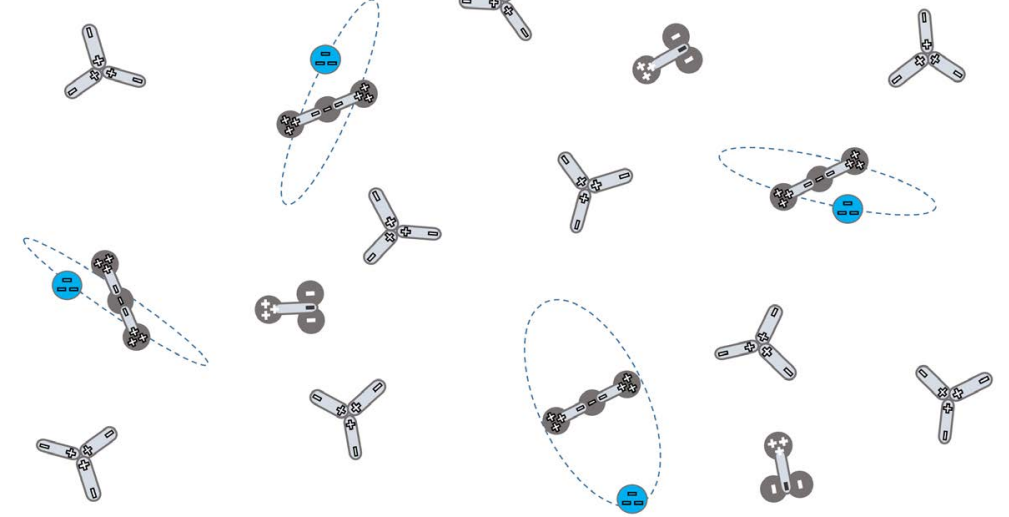

Figure 35. Universe filled with hydrogen atoms and rest neutrons and TSLs.

\section{Dark Matter Consideration}

The dark matter postulated from cosmological research is still outside the experimental range of our detection technologies. TSL in the YY model would be a candidate, or it isclosely related to dark matter.

Triple space links TSLs, as a released product of Triple Space Linked State (TSLS, Section 14.1), form "low-state" or "reaction-lazy" stable matter. They are postulated to have a small mass unit and, more importantly, to be charge neutral. They are the initial structure for the formation of matter. High energetic concentrations force them to build up and down quarks or to combine with them for producing real matter. They are also the product of electron-positron annihilation, as described in Section 9, accompanied by gamma rays. As shown in previous sections, they are released during some nuclear fusion and fission processes (Figure 27 and Figure 28). They can be consumed by other fusion processes (Figure 24 and Figure 26).

Apparently, free TSLs are lost outside our current observation and detection technologies. Bounded TSLs inside of atomic nuclei are interpreted by existing theories of interactions. It is possible to model their occurrence and consumption during the nuclear transformation and annihilation processes. Their role in the construction of the nucleus and their number are known. It should be possible to find some experimental methods that could verify the physical existence of TSL (the Y-Particle) and its mass unit by balancing all measurable masses and energies.

\section{Conclusions and Outlook}

The YY model opens up a broad spectrum of theoretical and experimental research in atomic physics and molecular chemistry. On the basis of the detailed construction structure of the atomic nucleus and its variants, electrostatic and quantum field calculations could deliver more filigree results than is currently possible. For example, how is the three-dimensional structure of a heavy atomic nucleus contracted in some parts and stretched or folded in other parts by elec- 
trostatic interactions between protonheads and neutronheads (both negatively charged) and protonides (positively charged).

So far, the governing forces for the PSL and TSL are hypotheses. A possible way to give them a basis is to combine them with QCD interpretation. Alternatively, similar methods from QCD can be used to clarify the necessary interactions in the YY model. The idea that the particle interactions are based on the cyclic groups Z2 (charges, materialized by the PSLs) and Z3 (colors, materialized by the TSLs) could be derived from this model for future analyses (Ref. [19]).

The distribution of electron clouds in orbitals around the atomic nucleus will also be important. A more detailed atomic model will reveal more details of the orbitals as well as molecular-level structures that have been hidden so far.

An isotope of an atom can have different nuclear structure configurations, as already shown for tritium H-3 (Figure 11) and for helium He-4 (Figure 13, Figure 16 and Figure 17). The search for differences in physical and chemical properties can reveal interesting aspects and contribute to the research of radioactive decay processes.

The possible relationship of the YY model to the existing preon model (Ref. [20] and [21]) is an open question. In the preon model, the compositeness as substructure is suggested to go beyond the Standard Model.

\section{Conflicts of Interest}

The authors declare no conflicts of interest regarding the publication of this paper.

\section{References}

[1] Gell-Mann, M. (1964) Physics Letters, 8, 214. https://doi.org/10.1016/S0031-9163(64)92001-3

[2] Gaillard, M.K., Grannis, P.D. and Scuilli, F.J. (1999) Reviews of Modern Physics, 71, S96. https://doi.org/10.1103/RevModPhys.71.S96

[3] Bortz, F. (2004) The Quark. The Rosen Publishing Group, Inc., New York.

[4] Epelbaum, E., Hammer, H.W. and Meißner, U.G. (2009) Reviews of Modern Physics, 81, 1773-1825. https://doi.org/10.1103/RevModPhys.81.1773

[5] Machleidt, R., Holinde, K. and Elster, C. (1987) Physics Reports, 149, 1-89. https://doi.org/10.1016/S0370-1573(87)80002-9

[6] Adamczyk, L., Adkins, J., Agakishiev, G., et al. (2015) Nature, 527, 345-348. https://doi.org/10.1038/nature15724

[7] Burkert, V.D., Elouadrhiri, L. and Girod, F.X. (2018) Nature, 557, 396-399. https://doi.org/10.1038/s41586-018-0060-Z

[8] Chu, J. (2019) Study of Quark Speeds Finds a Solution for a 35-Year Physics Mystery. MIT News Office.

[9] Schmookler, B., Duer, M., Schmidt, A., et al. (2019) Nature, 566, 354-358. https://doi.org/10.1038/s41586-019-0925-9

[10] Schmidt, A., Pybus, J., Weiss, R., et al. (2020) Nature, 578, 540-544. https://doi.org/10.1038/s41586-020-2021-6 
[11] Povh, B., Rith, K., Scholz, C., Zetsche, F. and Rodejohann, W. (2014) Particles and Nuclei. 9th Edition, Springer, Berlin. https://doi.org/10.1007/978-3-662-46321-5

[12] Ellwanger, U. (2012) From the Universe to the Elementary Particles. Springer, Berlin. https://doi.org/10.1007/978-3-642-24375-2

[13] Basdevant, J.-L., Rich, J. and Spiro, M. (2005) Fundamentals in Nuclear Physics-From Nuclear Structure to Cosmology. Springer, Berlin.

[14] Perkins, D.H. (2000) Introduction to High Energy Physics. 4th Edition, Cambridge University Press, Cambridge. https://doi.org/10.1017/CBO9780511809040

[15] Philips, A.C. (1999) The Physics of Stars. 2nd Edition, John Wiley \& Sons, Hoboken.

[16] Morse, E. (2018) Nuclear Fusion. Springer, Berlin. https://doi.org/10.1007/978-3-319-98171-0

[17] Huizenga, J.R. (1992) Cold Fusion: The Scientific Fiasco of the Century. University of Rochester Press, Rochester.

[18] Allday, J. (2001) Quarks, Leptons and the Big Bang. 2nd Edition, CRC Press, Boca Raton. https://doi.org/10.1201/9780585312354

[19] Abbas, S.A. (2017) Group Theory in Particle, Nuclear, and Hydron Physics. CRC Press, Boca Raton. https://doi.org/10.1201/9781315371702

[20] Dugne, J.J., Fredriksson, S. and Hansson, J. (2002) Europhysics Letters, 57, 188. https://doi.org/10.1209/epl/i2002-00337-8

[21] Fredriksson, S. (2004) Preon Prophecies by the Standard Model. Proceedings of the Fourth Tegernsee International Conference on Particle Physics beyond the Standard Model, Tegernsee, 9-14 June 2003, 211-223.

https://doi.org/10.1007/978-3-642-18534-2 13 